\title{
PreMBA Analytical Primer
}




\section{PreMBA Analytical Primer}

Essential Quantitative Concepts for Business Math

Regina Treviño 
PREMBA ANALYTICAL PRIMER

Copyright (๐) Regina Treviño, 2008.

Softcover reprint of the hardcover 1st edition 2008 978-0-230-60912-9

All rights reserved.

First published in 2008 by

PALGRAVE MACMILLAN ${ }^{\circledR}$

in the United States-a division of St. Martin's Press LLC,

175 Fifth Avenue, New York, NY 10010.

Where this book is distributed in the UK, Europe and the rest of the world, this is by Palgrave Macmillan, a division of Macmillan Publishers Limited, registered in England, company number 785998, of Houndmills,

Basingstoke, Hampshire RG21 6XS.

Palgrave Macmillan is the global academic imprint of the above companies and has companies and representatives throughout the world.

Palgrave ${ }^{\circledR}$ and Macmillan ${ }^{\circledR}$ are registered trademarks in the United States, the United Kingdom, Europe and other countries.

\section{ISBN 978-0-230-60913-6 ISBN 978-0-230-61578-6 (eBook) \\ DOI $10.1057 / 9780230615786$}

Library of Congress Cataloging-in-Publication Data

Trevino, Regina.

PreMBA analytical primer : essential quantitative concepts for business math / Regina Trevino.

p. $\mathrm{cm}$.

Includes bibliographical references and index.

1. Business mathematics. 2. Investments-Mathematics. I. Title.

HF5691.T74 2008

$650.01^{\prime} 513-d c 22$

2008006209

A catalogue record of the book is available from the British Library.

Design by Newgen Imaging Systems (P) Ltd., Chennai, India.

First edition: October 2008

109876554321 
a mi esposo Paul 


\section{CONTENTS}

Preface - xi

Mathematical Symbols and Abbreviations xiii

1 Business Math: Functions and Graphs 1

1.1 Functions 1

Application 1.1 The Revenue Function 2

1.1.1 Multivariate Functions 3

Application 1.2 The Demand and Supply Functions 3

1.2 Graphing Functions 4

Application 1.3 The Demand Curve 5

$\begin{array}{lll}1.3 & \text { Equations } & 7\end{array}$

$\begin{array}{lll}1.4 & \text { Linear Functions } & 7\end{array}$

1.4.1 Solving Linear Equations $\quad 8$

Application 1.4 Linear Demand Curves 9

1.4.2 Solving Linear Inequalities 10

$\begin{array}{lll}1.5 & \text { Slopes and Intercepts of Linear Equations } & 12\end{array}$

1.5.1 Slope-Intercept Form of a Line 13

Application 1.5 Graphing a Linear Demand Curve 17

1.5.2 Finding the Equation of a Line 19

1.5.3 General Linear Form of a Line 20

Application 1.6 The Consumer's Budget Constraint 21

1.5.4 Horizontal and Vertical Lines $\quad 25$

1.6 Solving Two-Variable Systems of Linear Equations 26

Application 1.7 Market Equilibrium $\quad 27$

$\begin{array}{lll}1.7 & \text { Properties of Functions } & 30\end{array}$

Application 1.8 Kinked Budget Constraints 31

1.8 Exponents 33

Application 1.9 Cobb-Douglas Production Function 33

1.8.1 Radicals and Fractional Exponents 34

1.8.2 Homogenous Functions $\quad 35$

Application 1.10 Constant Returns to Scale 35

1.9 Changes and Growth Rates 36

1.9.1 Growth Rate Formulas $\quad 37$

Application 1.11 The Sources of Economic Growth 38 
1.9.2 Index Numbers

Application 1.12 Consumer Price Index and Inflation 39

Practice Exercises

2 Business Math: Optimization $\quad 45$

2.1 Nonlinear Functions $\quad 45$

2.2 The Concept of a Derivative 45

2.2.1 Notation of Derivatives 47

2.2.2 Rules of Differentiation 47

Application 2.1 Marginal Functions 49

2.3 Graphical Interpretation of Derivatives $\quad 50$

Application 2.2 Marginal Product of Labor $\quad 52$

2.4 Additional Topics on Derivatives 54

2.5 Second Derivatives $\quad 55$

Application 2.3 Diminishing Marginal Utility $\quad 57$

2.6 Partial Derivatives $\quad 59$

Application 2.4 Partial Derivatives and the Ceteris

Paribus Assumption $\quad 60$

Application 2.5 Income Elasticity of Demand 60

2.7 Optimization 61

2.7.1 Unconstrained Optimization 62

Application 2.6 Profit Maximization 64

2.7.2 Constrained Optimization 66

Application 2.7 Consumer's Utility Maximization Problem 67

2.8 Exponential and Logarithmic Functions 69

Application 2.8 Continuously Compounded Interest Rate $\quad 70$

2.8.1 Logarithmic Functions 71

2.8.2 The Natural Logarithm 71

Application 2.9 Doubling Time for an Investment $\quad 72$

2.8.3 Differentiation of $e$ and $\ln \quad 73$

2.9 Measuring Areas under Linear Functions 73

Application 2.10 Consumer Surplus $\quad 74$

2.10 Economic Models 76

2.10.1 Flow and Stock Variables $\quad 77$

Application 2.11 Balance Sheet and Income Statement $\quad 77$

2.10.2 Nominal and Real Economic Variables $\quad 77$

Application 2.12 Nominal and Real GDP 77

2.11 Comparative Statics Analysis $\quad 79$

Application 2.13 Shifting the Demand and Supply Curves 79

Practice Exercises $\quad 81$

3 Statistical Analysis Primer $\quad 83$

3.1 Elements of Statistics $\quad 83$

3.2 Sigma Notation $\quad 84$ 
3.3 Measures of Central Tendency 85

3.4 Frequency Tables and Histograms 87

3.5 Measures of Variability 92

3.6 Measures of Association 93

3.7 Probability Essentials 97

3.8 Discrete Random Variables 98

3.8.1 Discrete Random Variables and Expected Value $\quad 100$

3.8.2 Discrete Random Variables and Variance 101

3.8.3 Decision Tree Basics 103

3.9 Continuous Random Variables 106

3.9.1 The Normal Distribution 107

3.9.2 Standardizing: $\mathrm{z}$-Values 108

3.9.3 Standard Normal Tables and z-Values 108

3.10 An Introduction to Estimation 112

3.10.1 The Sampling Distribution of the Sample Mean 113

3.11 Regression Analysis $\quad 115$

3.11.1 Simple Linear Regression 115

3.12 Hypothesis Testing 121

3.12.1 Types of Errors $\quad 121$

3.12.2 Significance from $\mathrm{p}$-Values 122

Practice Exercises 124

4 Mathematics of Finance 131

4.1 Time Value of Money 131

4.1.1 Present Value 132

4.1.2 Future Value 133

4.2 Nominal and Real Rates of Interest 134

4.3 Compound Interest 135

4.3.1 Continuous Compounding 136

4.3.2 Effective Interest Rate 137

4.4 Annuities 139

4.4.1 Amortization of Loans 139

4.5 Perpetuities 140

4.5.1 Growing Perpetuities 142

4.6 Net Present Value 143

4.6.1 Discounted Cash Flow 144

4.6.2 Risk-Adjusted Discount Rates 145

4.7 Bonds 146

4.7.1 Zero-Coupon Bonds 148

4.8 Common Stocks 149

4.9 Portfolio Theory Primer 150 
CONTENTS

4.9.1 Portfolio Math

150

4.9.2 Diversification and Portfolio Risk

154

4.9.3 Measuring Risk

155

Practice Exercises

158

Appendix A: List of Rules 163

Appendix B: Answers to Practice Exercises 169

Notes 193

$\begin{array}{ll}\text { References } & 197\end{array}$

$\begin{array}{ll}\text { Index } & 199\end{array}$ 


\section{PREFACE}

This book is a review of the analytical methods required in most of the quantitative courses taught at MBA programs. Students with no technical background, or who have not studied mathematics since college, may easily feel overwhelmed by the mathematical formalism that is typical of quantitative courses, such as economics, statistics, or finance. These students will benefit from a concise and focused review of the analytical tools that will become a necessary skill in their MBA classes. The objective of this book is to present the essential quantitative concepts and methods in a self-contained, nontechnical, and intuitive way.

I divide the contents of the book into three broad sections: business math, statistics, and finance. In the business math section (chapters 1 and 2), each analytical method is followed by an application that illustrates how such tool is typically used in MBA quantitative courses. In these applications, wherever possible, I provide the background and context to facilitate understanding and stimulate interest.

Chapter 3 is a statistical analysis primer and chapter 4 is a review of the mathematics of finance. The topics covered in both these chapters represent a knowledge basis that is essential before taking any MBA statistics, operations research, and finance courses. Simple examples are used to illustrate the topics covered in chapters 3 and 4 .

\section{The Style of The Book}

In choosing the contents of PreMBA Analytical Primer: Essential Quantitative Concepts for Business Math, I tried to err on the side of inclusion. My aim is to assure coverage of most of the analytical tools that students may be required to handle during their MBA courses.

I sought a style of presentation that is accessible yet rigorous. Wherever possible, I give precise definitions of the concepts covered. At the same time, I accompany this analysis with examples that illustrate the key points.

Topics that I consider either relatively advanced or too peripheral are marked with an asterisk (*). This allows students to skip them over easily in a first reading. 


\section{How To Use This Book}

This book is intended to be a math refresher that serves students both as a primer, and as a reference along their courses. Students with little technical background will benefit from reading and working on the entire book before the start of their MBA classes. Others students, however, might use it as a reference tool for the review of particular topics on a need basis throughout their MBA courses.

The chapters have been written to be relatively self-contained. As a result, a student can alter the sequence in which the different topics (math, statistics, and finance) are reviewed. At the end of the book, I provide a listing with all the major rules covered in the chapters and I also include a brief summary of some basic algebra rules.

I include exercises that test students' understanding of the topics throughout the text as well as a set of practice problems at the end of each chapter. Working through as many of these exercises as possible is the surest way to master the material covered in each chapter. The answers to all the test-yourunderstanding exercises and practice problems are included at the end of the book.

\section{AcKnowledgments}

I am very grateful for the constructive comments, advice, and support from my colleagues and friends Bo Becker, Luis Garicano, Tapio Pekkala, Luis Rayo, Lars Stole, and especially, Mine Cinar. I would also like to acknowledge the efforts of my students Dong Li and Jerry Wang, who proofread the entire manuscript.

It was a pleasure to work with everyone at Palgrave Macmillan. My acquisitions editor, Laurie Harting, was a source of excellent advice. Erin Ivy, my production editor, did a sterling job and so did Maran Elancheran and his crew at New Imaging Systems.

Finally, I would like to thank my family, especially my husband Paul, for their loving support and patience during the late nights that I spent working on this project. 


\section{Mathematical Symbols and ABBREVIATIONS}

The use of symbols and notation is explained in a self-contained discussion wherever these appear in each chapter, but for convenience I also list here the major symbol conventions that recur throughout the book.

\section{Symbol Meaning}

$\Delta \quad$ Greek letter delta. Denotes change in the value of a variable.

$\Pi \quad$ Greek letter pi (uppercase). Denotes firm profits.

$\Sigma \quad$ Greek letter sigma (uppercase). Denotes summation.

$\alpha \quad$ Greek letter alpha. Denotes significance level of a test.

$\beta \quad$ Greek letter beta. Denotes sensitivity of a security's return to market movements.

$\varepsilon \quad$ Greek letter epsilon. Denotes elasticity (chapter 2). Denotes random error (chapter 3).

$\lambda \quad$ Greek letter lambda. Denotes Lagrange multiplier.

$\pi \quad$ Greek letter pi (lowercase). Denotes inflation.

$\mu \quad$ Greek letter mu. Denotes mean.

$\rho \quad$ Greek letter rho. Denotes correlation coefficient.

$\sigma \quad$ Greek letter sigma (lowercase). Denotes standard deviation.

A Generic constant. In a Cobb-Douglas production function, it denotes factor productivity.

a Generic constant. Typically used to denote the vertical intercept of a line.

$b \quad$ Generic constant. Typically used to denote the slope of a line.

C Generic constant. Denotes cash flow (chapter 4).

$C(\cdot) \quad$ Cost function.

cdf Cumulative distribution function.

CPI Consumer price index.

CS Consumer surplus.

d Generic constant. Next to a variable, it denotes small (infinitesimal) change in the value of the variable.

DCF Discounted cash flow.

DIV Dividend.

$E(\cdot) \quad$ Expected value.

$f(\cdot) \quad$ Function. 
$f^{\prime}(\cdot) \quad$ First derivative of the function.

$f^{\prime \prime}(\cdot)$ Second derivative of the function.

$F \quad$ Face value.

FOC First order condition.

FV Future value.

I Consumer income.

g Growth rate.

GDP Gross domestic product.

$K \quad$ Capital stock.

$L \quad$ Labor.

In Natural logarithm.

$M C$ Marginal cost.

$M P L \quad$ Marginal product of labor.

$M U$ Marginal utility.

$N \quad$ Normal distribution.

NPV Net present value.

$p \quad$ Price of a good.

$p_{a} \quad$ Price of an alternative good.

$p_{m} \quad$ Price of the materials used to produce a good.

$p_{t} \quad$ Price of technologically related goods.

$P \quad$ Principal.

pdf Probability density function.

PV Present value.

$q \quad$ Quantity (individual level).

$Q \quad$ Quantity (market level)

$Q^{d} \quad$ Quantity demanded.

$Q^{s} \quad$ Quantity supplied.

$R \quad$ Firm revenue.

$r \quad$ Interest rate.

$r_{e} \quad$ Effective interest rate.

$r_{f} \quad$ Risk-free interest rate.

$r_{x y} \quad$ Sample correlation coefficient between variable $x$ and variable $y$.

$s \quad$ Sample standard deviation.

SOC Second order condition.

$t \quad$ Index variable for time (chapter 4).

$U(\cdot) \quad$ Utility function.

$x \quad$ Generic variable. Typically denotes the independent variable.

$\bar{x} \quad$ Sample mean.

$y \quad$ Generic variable. Typically denotes the dependent variable.

$\Upsilon \quad$ Generic variable. In the aggregate production function, it denotes total output. 
II Absolute value.

$\infty$ Infinity.

a Partial derivative.

L(·) Lagrangian.

\section{Asterisks, Hats, and Primes}

Asterisks: An asterisk is attached to a variable to denote optimal, equilibrium, or solution quantity (e.g., $x^{*}$ ).

Hats: A hat is attached to a variable to denote predicted value (e.g., $\hat{y}$ ).

Primes: A prime attached to a function of a single variable denotes first derivative (e.g., $\left.f^{\prime}(x)\right)$.

A prime attached to a variable, as opposed to a function, denotes a specific value of that variable (e.g., $\left.p^{\prime}\right)$. 